\title{
Some effects of fixed-ratio slze and reinforcement frequency on the accuracy of a conditional discrimination performance'
}

JOHN J. RANDOLPH, Abbott Laboratories, North Chicago, III. 60064

The effects of two values of fixed-ratio size, FR 1 and FR 5 , and two frequencies of reinforcement, one pellet and five pellets, on the accuracy of a conditional discrimination were studied in the baboon. In general, no differences in accuracy were observed when either $F R 1$, one pellet or FR 5, five pellets were used. High levels of accuracy were produced by the FR 5, one pellet reinforcement contingency.

Ferster (1960), Ferster \& Appel (1961), and Zimmerman \& Ferster (1963) have reported that fixed-ratio reinforcement and timeout (TO) from positive reinforcement control error levels in a matching-to-sample performance. Ferster \& Appel (1961) concluded that this control was a function of reinforcement frequency. This experiment was designed to confirm this point within the context of fixed-ratio schedules. The discrimination of two fixed-ratio sizes (Rilling \& McDiarmid, 1965; Pliskoff \& Goldiamond, 1966) was used as the conditional discrimination performance. The number of pellets delivered to the $S$ was varied as a function of the number of correct responses required to produce reinforcement.

\section{METHOD}

The S, an adult female baboon (Papio papio), was experimentally naive at the beginning of the investigation.

The experiment was conducted in the multi-animal environment with separate entrance cubicles described by Ferster, Hammer, \& Randolph (1968). The animal was assigned to one of the small work cubicles. A work panel and a feeder panel were located on one wall of the chamber. Three stimulus projection units, spaced 3 in. apart, were mounted on the work panel. A Lev-R switch was located 1 in. below each of the stimulus projection units. A feeder was placed behind an adjacent panel. When the pilot light on the feeder panel was on, along with a tone, each response on an associated Lev- $R$ switch delivered one pellet to the $S$.

The contingencies were programmed with relays and clocks, and the data were recorded on electromechanical counters and a cumulative recorder. The general performances demanded by the multi-animal environment were acquired under the procedures described by Ferster, Hammer, \& Randolph (1968). The discrimination of the two fixed-ratio (FR) requirements was then developed in small steps.

During the initial stages of training, either 5 or 25 responses on the center switch, when the center projection unit was on, turned that unit off and illuminated the projection units on each side. If the larger of the two response requirements had been programmed on the center switch, $p=0.50$, a response on the right switch was correct. A response on the left-hand key was correct if the smaller of the two response requirements had been programmed on the center switch. Incorrect responses and responses on a switch which was not associated with an illuminated projection unit were followed by a 5 -sec TO. During the TO none of the projection units was illuminated, a low-frequency buzzer was turned on, and responses on any of the switches reset the 5-sec clock. A correction procedure was employed to prevent the development of position preferences, i.e., the fixed ratio programmed on the center key was not subject to change following an error. Correct responses turned the feeder panel on and the $S$ was allowed to operate it once.

Water was available at all times. $A$ tube and nipple, connected to a watering system, were located to the right of the work panel. An electric valve was opened for the duration of the response on the water switch. To prevent this behavior from becoming an adventitious member of the discriminative performance, each response on the water switch initiated a TO which ended $15 \mathrm{sec}$ after the response was over. The low-frequency buzzer was not present during this TO.

The size of the larger of the two fixed ratios was gradually reduced over a period of several weeks to FR 8 . When the performance was stable, the number of correct responses which preceded the opportunity to operate the feeder and the number of times the $S$ was allowed to operate the feeder were manipulated systematically. Correct responses which were not reinforced with food produced a .75-sec presentation of the stimuli which normally accomplished the opportunity to operate the feeder. Each of these conditions was left in effect until a stable level of performance had been achieved. The discrimination of the two fixed-ratio sizes was reinforced on one of two fixed ratios, FR 1 or FR S, and the reinforcement frequency was either one pellet or five pellets. The combinations of these parameters, the length of time they were in effect, and the order in which they were presented to the $S$ are given in Table 1.

Sessions were terminated after a fixed number of pellets was given. The $S$ received $60(.750-g)$ pellets per session until the second presentation of FR 1 and one pellet. At this time the number of pellets per session was reduced to $45(1.0-\mathrm{g})$ pellets. At the end of the session the $S$ was allowed to go from the work cubicle to the social area for the 2-h intersession interval. The $S$ could return to the work cubicle at any time following the intersession interval. With these exceptions the experiment ran continuously, and the $S$ worked $4-8$ sessions per day $(\mathrm{mdn}=6)$.

\section{RESULTS AND DISCUSSION}

The results of the experiment are shown in Fig. 1. The initial error levels obtained with FR 1 , one pellet and FR 5 , five pellets are quite similar. These data suggest the simple requirement of multiple correct responses for reinforcement is not a sufficient condition for the reduction of errors.

At this point the $S$ had correctly solved the problem more than 18,000 times with no evidence of any reduction of errors beyond the first few sessions. However, when the

\section{Table 1}

The Combinations of Fixed Ratio Sizes, Reinforcement Frequency, and the Number of Days they were in Effect. They are given in Chronological Order.

\begin{tabular}{ccc}
\hline FIXED RATIO SIZE & NUMBER OF PELLETS & DAYS \\
FR 1 & 1 & 17 \\
FR 5 & 5 & 34 \\
FR 5 & 1 & 13 \\
FR 5 & 5 & 11 \\
FR 1 & 1 & 12 \\
FR 5 & 5 & 18 \\
FR 5 & 1 & 18 \\
FR 1 & 1 & 20 \\
FR 5 & 5 & 29 \\
FR 1 & 1 & 21 \\
\hline
\end{tabular}




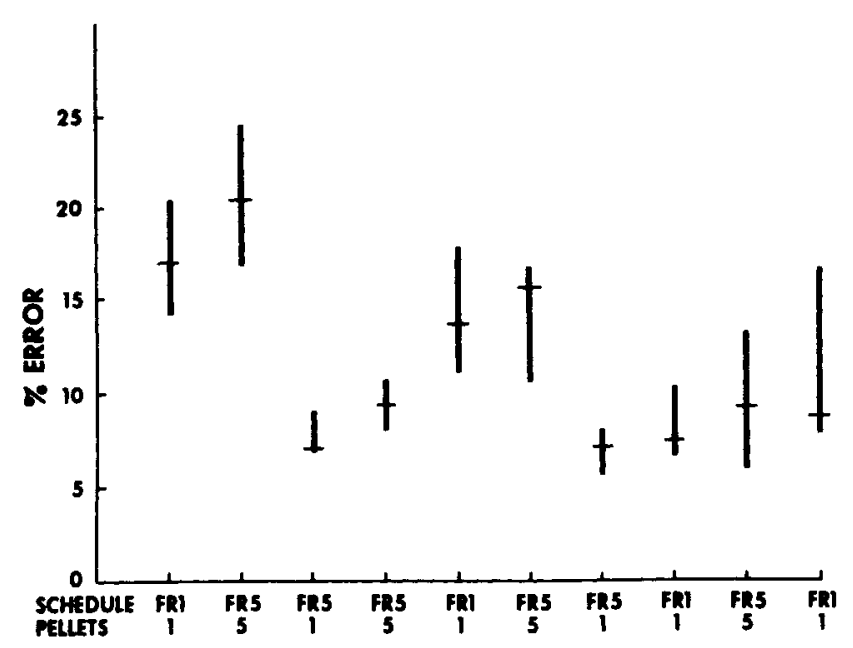

Fig. 1. Relative frequency of errors as a function of ratio size and number of pellets. Each point is the median and range of the last four days under each condition of the experiment.

reinforcement frequency was reduced to one pellet for every five problems solved correctly, errors decreased rapidly. A return to FR 5, five pellets and FR 1, one pellet resulted in an increase in the error level. Error levels again decreased when one pellet was delivered for five correct problems.

The third determination of error levels with FR 1, one pellet and FR 5, five pellets produced a marginal, if any, increase in error level. The error levels produced by FR 5, five pellets and FR 1, one pellet also appear to be influenced by their temporal proximity to FR 5 , one pellet.
The data confirm the suggestion by Ferster (1960) and Ferster \& Appel (1961) that reinforcement frequency is one of the critical determinants of the accuracy of conditional discrimination performances. The apparent induction from FR 5, one pellet to the conditions immediately following it resembles the induction effects reported between fixed-ratio and fixed-interval schedules by Ferster (1960).

Further development of this kind of procedure may provide a vehicle for the study of transition states from higher to lower error levels. Excluding the points determined immediately after FR 5, one pellet, an orderly decrease in error level is seen as a function of the introduction of FR 5, one pellet. Therefore, a transition state could presumably be started after the $S$ had been thoroughly adapted to the environment and had learned all of the basic elements of the experimental repertoire. This might eliminate control by a number of extraneous variables which complicate the study of transition states in "naive" organisms.

\section{REFERENCES}

FERSTER, C. B. Intermittent reinforcement of matching to sample in the pigeon. Journal of the Experimental Analysis of Behavior, 1960, 3, 259-272.

FERSTER, C. B., \& APPEL, J. B. Punishment of $\mathrm{S}^{\Delta}$ responding in matching to sample by time out from positive reinforcement. Journal of the Experimental Analysis of Behavior, 1961, 4, 45-56.

FERSTER, C. B., HAMMER, C., \& RANDOLPH, J. An experimental space combining individual and social performances. Journal of the Experimental Analysis of Behavior, 1968, 11, 209-220.

PLISKOFF, S. S., \& GOLDIAMOND, I. Some discriminative properties of fixed ratio performance in the pigeon. Joumal of the Experimental Analysis of Behavior, 1966, 9, 1-9.

RILLING, M., \& McDIARMID, C. Signal detection in fixed-ratio schedules. Science, 1965, 148, 526-527. NOTE

1. This research was supported by National Grant NsG-450 to the Institute for Behavioral Research. The author gratefully acknowledges the editorial assistance of Sue Bocknik. 